\title{
Monotone heteroclinic solutions to non-autonomous equations via phase plane analysis
}

\author{
Andrea Gavioli
}

\begin{abstract}
We study the existence of at least one increasing heteroclinic solution to a scalar equation of the kind $\ddot{x}=a(t) V^{\prime}(x)$, where $V$ is a non-negative double well potential, and $a(t)$ is a positive, measurable coefficient. We first provide with a complete answer in the definitively autonomous case, when $a(t)$ takes a constant value $l$ outside a bounded interval. Then we consider the case in which $a(t)$ is definitively monotone, converges from above, as $t \rightarrow \pm \infty$, to two positive limits $l_{*}$ and $l^{*}$, and never goes below $\min \left(l_{*}, l^{*}\right)$. Furthermore, the convergence to $\max \left(l_{*}, l^{*}\right)$ is supposed to be not too fast (slower than a suitable exponential term).
\end{abstract}

Mathematics Subject Classification (2000). 34C37.

Keywords. Heteroclinic, Double-well potential, Hamiltonian flow, Measure-preserving, Shooting method.

\section{Introduction}

The existence of heteroclinic solutions to a variational equation of the kind $\ddot{x}=W_{x}^{\prime}(t, x)$ was widely studied in the case in which $W(t, x)$ is periodic or almost periodic with respect to $t$ (see, for instance $[10,11]$ ). On the contrary, at our knowledge, rather few results are available under assumptions of different nature: for instance, we refer to [4] (Sect. 5, Example 1) for the case in which $W(t, x)$ is definitively monotone with respect to $t$, and to [2] (Chapter 2, Thm. 2.2) for equations of the kind

$$
\ddot{x}=a(t) V^{\prime}(x),
$$

where $V$ is a double well potential, the coefficient $a(t)>0$ converges to a positive limit $l$ as $|t|$ diverges and fulfils the inequality $a(t) \leq l$ everywhere: in 
this case, the authors prove that a solution always exists, which also minimizes the associated integral functional. On the contrary, when $a(t) \geq l$, it looks difficult to get an exhaustive answer, and even more so when $a(t)-l$ changes its sign. In [5] we began to tackle the case $a(t) \geq l$ by means of minimax methods, and proved the existence of a solution under the technical assumption that the ratio $a(t) / l$ is bounded from above by a constant which depends on the shape of $V$ (see also [12] for a recent improvement of this result). In [6], where we employ perturbative methods, we do not need this bound, but we require that $a(t)$ is definitively monotone.

In the present paper we are going to introduce further techniques for tackling the problem. As explained by the title, they arise from the study of the first order system which corresponds to (1.1):

$$
\dot{u}=f(t, u), \quad u=(x, y) \in \mathbb{R}^{2},
$$

where $f(t, x, y)=\left(y, a(t) V^{\prime}(x)\right)$. For the sake of simplicity, we suppose that the two wells of $V$ are located at \pm 1 , so that (1.2) presents two saddle points at $( \pm 1,0)$. Our aim is to solve problem $(2.1)$, that is to find an increasing, heteroclinic solution of (1.1) which connects the two unstable equilibria \pm 1 . On this subject, the two main results are Theorems 2.4 and 5.1, which are proved by means of fairly different techniques: in the former, indeed, we deal with the "definitively autonomous" case (2.2), and exploit the well-known property that a hamiltonian flow preserves area. In the latter, on the contrary, $a(t)$ converges to two possibly different limits $l_{*}$ and $l^{*}$ as $t$ diverges, respectively, to $-\infty$ and $+\infty$, and the proof is based on a shooting method.

The plan of the work is conceived as follows: in Sect. 2 we first state Theorem 2.4, and present some preliminary results. In particular, we explain some properties of measure-preserving diffeormorphisms in the plane, which are summed up in Lemma 2.7. The proof of the theorem is based on the following, simple idea: the heteroclinic trajectory of the autonomous version of (1.2) which joins $( \pm 1,0)$ in the half-plane $y>0$ is compelled to meet its image through the hamiltonian flow, because the differential form $\int y d x$ is shown to be preserved, in this framework. Actually, the search for monotone solutions gives rise to some difficulties: in order to tackle them, indeed, we need a technical result (Lemma 2.5) which is proved in Sect. 6. As far as we are only interested in $(2.1 \mathrm{a}-\mathrm{b})$, however, the proof becomes more simple, and condition (ii) can be replaced by other assumptions (Theorem 2.10).

In Sect. 3 we begin to explain our shooting method, where the two equilibria $(-1,0)$ and $(1,0)$ of $(1.2)$ act respectively as the starting point and the target: roughly speaking, we consider the family of all solutions $x$ of (1.1) such that $x(-\infty)=-1$, and put in evidence, at the beginning of the section, the two cases $\left(H^{-}\right)$and $\left(H^{+}\right)$, which correspond to disjoint open sets $C^{-}$and $C^{+}$ in the projection of the unstable manifold of $(-1,0)$ on the plane $t x$. If we are able to prove that both cases occur, then a connectedness argument shows that they cannot be exhaustive, so that some function $x$ of the family should converge to 1 , or to some critical point of $V$ between \pm 1 : since, however, the 
latter behaviour is ruled out by condition (ii) of Sect. 2, we should actually get $x(+\infty)=1$.

In Sect. 4 we perform a detailed study of (1.1) near the well $x=-1$, also by means of variational methods. In particular, the unstable manifold of (1.2) in the neighbourhood of $(-1,0)$ is represented in the form $\eta=\eta(\tau, h)$, where $\tau,-1+h$ and $\eta$ stand for time, position and speed respectively. This function $\eta$ allows to outline correctly our shooting method: in particular, its continuity shows that both sets $C^{-}$and $C^{+}$are actually open (Lemma 3.3). Furthermore, other useful properties of $\eta$ are explained, some of which are due to the particular conditions that the coefficient $a(t)$ is supposed to satisfy in Theorem 5.1. The section ends with a study of the functions $(3.4,3.5)$ and some related estimates.

In Sect. 5 we accomplish our shooting method, by showing that the two sets $C^{-}$and $C^{+}$we introduced before are actually non-empty, so as to prove Theorem 5.1, that is the existence of solutions to (2.1) under the following assumptions: $a(t)$ converges from above to $l_{*}$ and $l^{*}$, is definitively monotone and never goes below $\min \left(l_{*}, l^{*}\right)$. Furthermore, the convergence to $\max \left(l_{*}, l^{*}\right)$ is not too fast, since we require an exponential bound on the speed of that convergence, which is given by (5.1) or (5.2). We point out that this condition recalls very closely the one which appears in $[7,8]$ (Thm. 1.7). The paper ends with the already quoted Sect. 6 , which is an appendix to Sect. 3 .

With respect to the results of $[5,6,12]$, the main advantage of Theorem 5.1 is the possibility to deal with different limits $l_{*}, l^{*}$. Furthermore, as regards a comparison with $[5,12]$, it needs no bounds from above on the coefficient $a(t)$. On the other hand, in $[5,12]$ no conditions are required on the convergence of $a(t)$ to $l$, and also the condition (ii) on the potential $V$ can be dropped. In order to conclude, we remark that the assumptions which are needed in the different approaches of $[5,6,12]$ and the present paper do not cover all possible cases: nevertheless, at our knowledge, no counter-example to the existence of heteroclinic solutions to (1.1) was found yet, when $l^{*}=l_{*}$. On the contrary, in the case $l^{*} \neq l_{*}$, several counter-examples can be produced (see Remarks 3.6 and 5.5).

\section{The definitively autonomous case}

We are interested in the following problem:

$$
\begin{cases}\ddot{x}(t)=a(t) V^{\prime}(x(t)) & (a) \\ x(-\infty)=-1, x(+\infty)=1 & (b) \\ \dot{x}>0 & (c)\end{cases}
$$

Throughout this section we assume that:

(i) $V \in C^{2}\left(\mathbb{R} ;\left[0,+\infty[), V( \pm 1)=0, V^{\prime \prime}( \pm 1)>0\right.\right.$.

(ii) $V^{\prime}$ vanishes at just one point $\left.x_{0} \in\right]-1,1$, where $V^{\prime \prime}\left(x_{0}\right)<0$.

(iii) $a \in L^{\infty}(\mathbb{R} ;[0,+\infty[)$.

(iv) There exist $l, S, T \in \mathbb{R}$ such that $l>0, S<T$, and 


$$
a(t)=l \quad \text { for } \quad t \notin[S, T] .
$$

Conditions (i) and (ii) are shared by some of the most common double well potentials. In particular, they entail the strict inequality $V>0$ on ] $-1,1[$. Of course, it does not matter that the two wells are located at \pm 1 , as we supposed for the sake of simplicity.

Remark 2.1. Since the coefficient $a(t)$ is only supposed to be measurable, the solutions of (2.1a) are to be understood in the usual weak sense, that is to say: $x$ is $C^{1}, \dot{x}$ is absolutely continuous and (2.1a) holds almost everywhere (more precisely: at any Lebesgue point of $a)$.

Sometimes it will be useful to suppose that $V$ fulfils the further condition we are going to introduce below. Actually, as explained by the following remark, we are allowed to do it, without loss of generality:

$$
V^{\prime \prime}(x) \equiv V^{\prime \prime}( \pm 1) \quad \text { for } \quad|x| \geq 1 \text {. }
$$

Remark 2.2. In order to justify $(2.3)$, it is enough to modify $V$ outside $[-1,1]$, so as to get a new potential $V_{1}$ which fulfil (2.3). Indeed, put $V=V_{1}$ in (2.1), and suppose to have found a solution $x=x(t)$ of the corresponding problem: since $\dot{x}>0$, it is $-1<x(t)<1$ everywhere, so that $x$ solves the original problem as well. In particular (2.3) entails that $V^{\prime}$ has a linear growth, so that all solutions to (2.1a) are global.

Definition 2.3. We say that a field $f$ admits a linear domination if there exists a constant $C$ such that $\|f(t, u)\| \leq C(1+\|u\|)$ for $t \in \mathbb{R}, u \in \mathbb{R}^{2}$.

Theorem 2.4. Let (i)-(iv) hold. Then problem (2.1) admits a solution.

The proof of this result requires some preliminary arguments: first of all, in order to fulfil (2.1c), we need to modify the field (1.2) in a suitable way, since the technique we are going to adopt does not provide, in a natural way, with monotone solutions. To this end we introduce the following, technical result, whose proof is given in Sect. 6.

Lemma 2.5. Let $a$ and $V$ fulfil conditions (i)-(iii) and $f$ be as in (1.2): then there exists a hamiltonian field $f_{*}(t, x, y)$ such that $f_{*} \equiv f$ for $y \geq 0, f_{*}$ admits a linear domination, $f_{*}-f$ is autonomous and $C^{1}$, and the following property holds: whenever $u=(x, y)$ solves the equation $\dot{u}=f_{*}(t, u)$ and $u( \pm \infty)=$ $( \pm 1,0)$, it is $y>0$ everywhere.

According to the previous result, whenever we are able to find a solution $u=(x, y)$ of the equation $\dot{u}=f_{*}(t, u)$ which fulfils the conditions $u( \pm \infty)=$ $( \pm 1,0)$, then $x$ solves (2.1). On the other hand, for $t \notin[S, T]$ and $y \geq 0, f$ agrees with the autonomous field $f^{l}:(x, y) \mapsto\left(y, l V^{\prime}(x)\right)$. As is known, the conditions $-1<x<1, y=\sqrt{2 l V(x)}$ define, in the phase plane, a curve $\Gamma$ which is a heteroclinic trajectory for $f^{l}$, and joins together the two equilibria $( \pm 1,0)$. We can conclude that (2.1) surely admits a solution if so does the following problem:

$$
\begin{cases}\dot{u}(t)=f_{*}(t, u(t)) & (a) \\ u(S), u(T) \in \Gamma & (b)\end{cases}
$$


Remark 2.6. Since the field $f_{*}(t, u)$ is hamiltonian, its flow $\psi(t ; \sigma, w)$ enjoys the following property: for any $\sigma, t \in \mathbb{R}$, the map $w \mapsto \psi(t ; \sigma, w)$ preserves area. Indeed, even if $f_{*}(t, u)$ is only supposed to be measurable with respect to $t$, the same arguments as in Lemma 2.8 ensure that $\psi$ is continuously differentiable with respect to $w$, and the matrix $\nabla_{w} \psi(t ; \sigma, w)$ solves a well-known variational equation, from which we argue, by virtue of Liouville's Theorem, that its determinant is identically 1.

On the ground of the previous remark, we are going to explain some properties of measure preserving diffeomorphisms on $\mathbb{R}^{2}$, whose family we denote by $\mathcal{F}$. In particular, we are interested in the behaviour of the differential form $\int y d x$ under the action of a map $\phi \in \mathcal{F}$. To this end, we introduce the family $\mathcal{P}$ of all piece-wise regular arcs $\gamma: I \rightarrow \mathbb{R}^{2}$, where $I=[-1,1]$, and put $F(\gamma)=\int_{\gamma} y d x$ for any $\gamma \in \mathcal{P}$. We also define $\mathcal{P}^{*} \subseteq \mathcal{P}$ as the family of all simple, regular arcs $\gamma: I \rightarrow \mathbb{R}^{2}$ such that $\gamma( \pm 1)=( \pm 1,0)$, and $\mathcal{F}^{*} \subseteq \mathcal{F}$ through the conditions $\phi( \pm 1,0)=( \pm 1,0)$. Then we put, for any $\phi \in \mathcal{F}^{*}:$

$$
\Omega(\phi)=F(\phi \circ \gamma)-F(\gamma)
$$

where $\gamma \in \mathcal{P}^{*}$ is arbitrarily chosen. Such a definition is actually well-posed, since the right-hand side of (2.5) defines a closed differential form on the whole plane, as we can easily check through the identity det $\nabla \phi \equiv 1$. We also recall that, whenever $\gamma, \eta \in \mathcal{P}^{*}$, we can define a closed arc $\gamma * \eta^{-} \in \mathcal{P}$, where $\eta^{-}(s)=\eta(-s)$, and the symbol $*$ denotes the usual operation between consecutive paths.

Lemma 2.7. The following properties hold true.

(a) $\phi \circ \gamma \in \mathcal{P}^{*}$ for any $\phi \in \mathcal{F}^{*}, \gamma \in \mathcal{P}^{*}$.

(b) $\Omega\left(\phi_{n} \circ \cdots \circ \phi_{1}\right)=0$ whenever $\phi_{i} \in \mathcal{F}^{*}$ and $\Omega\left(\phi_{i}\right)=0(i=1, \ldots, n)$.

(c) $\Omega$ is continuous from $C_{\text {loc }}^{1}\left(\mathbb{R}^{2} ; \mathbb{R}^{2}\right)$ to $\mathbb{R}$.

(d) If $\phi(\chi(I))=\chi(I)$ for some $\chi \in \mathcal{P}^{*}$, then $\Omega(\phi)=0$.

(e) If $\Omega(\phi)=0$, then $\phi\left(\gamma\left(I^{\circ}\right)\right) \cap \gamma\left(I^{\circ}\right) \neq \emptyset$.

Proof. (a) Let $\gamma \in \mathcal{P}^{*}, \eta=\phi \circ \gamma$ : then $\phi( \pm 1,0)=( \pm 1,0) \Rightarrow \eta( \pm 1)=$ $( \pm 1,0)$. Since $\phi$ is a diffeomorhism, $\eta$ is simple and regular, like $\gamma$.

(b) The previous step entails that $\phi:=\phi_{n} \circ \cdots \circ \phi_{1} \in \mathcal{F}^{*}$. Now, let $\gamma_{0} \in \mathcal{P}^{*}$, and put $\gamma_{i}=\phi_{i} \circ \gamma_{i-1}$ for $i=1, \ldots, n$ : the equality $\Omega\left(\phi_{i}\right)=0$ implies $F\left(\gamma_{i}\right)=F\left(\gamma_{i-1}\right)$, so that $F\left(\phi \circ \gamma_{0}\right)=F\left(\gamma_{n}\right)=F\left(\gamma_{0}\right)$.

(c) Let $\phi \in \mathcal{F}^{*}$ : then $\Omega(\phi)=\int_{-1}^{1} \beta(s, 0) \alpha_{x}^{\prime}(s, 0) d s$, where $\alpha$ and $\beta$ are the components of $\phi$. Indeed, let us put $\bar{\gamma}(s)=(s, 0)$ for any $s \in I$, and apply $(2.5)$ to $\gamma=\bar{\gamma}$ : since $F(\bar{\gamma})=0$, we get $\Omega(\phi)=F(\phi(\bar{\gamma}))$, which actually yields the integral above. Now our assertion follows easily.

(d) Since $\phi(\chi(I))=\chi(I)$, the two regular $\operatorname{arcs} \chi$ and $\rho:=\phi \circ \chi$ have the same trace and direction, so that $F(\rho)=F(\chi)$. Hence the right-hand side of (2.5) vanishes, when putting $\gamma=\chi$.

(e) By contradiction, suppose that $\eta\left(I^{\circ}\right) \cap \gamma\left(I^{\circ}\right)=\emptyset$, where $\eta=\phi \circ \gamma$. Then $\gamma * \eta^{-}$is a Jordan arc, which encloses a regular domain with area $\lambda>0$. 
Now Green's formulas yield the contradiction $F\left(\gamma * \eta^{-}\right)=F(\gamma)-F(\eta)=$ $\pm \lambda \neq 0$.

The following result can be proved by the same techniques which allow to get, under suitable assumptions, the differentiability of a flow with respect to initial conditions, and the continuity of its jacobian matrix with respect to a parameter (see, for instance [1]).

Lemma 2.8. Let $\sigma, \tau \in \mathbb{R}, \sigma<\tau$. For any $n \in \mathbb{N}^{*}:=\mathbb{N} \cup\{\infty\}$ let $a_{n} \in$ $L^{\infty}([\sigma, \tau])$ be such that $a_{n} \rightarrow a_{\infty}$ in $L^{1}([\sigma, \tau])$ as $n \rightarrow+\infty$. Let $K<+\infty$ be the least upper bound of $\left\|a_{n}\right\|_{\infty}$ when $n$ ranges over $\mathbb{N}^{*}$. For any $n \in \mathbb{N}^{*}, u \in \mathbb{R}^{2}$, let us put $f_{n}(t, u)=a_{n}(t) p(u)+q(u)$, where $p, q \in C^{1}\left(\mathbb{R}^{2} ; \mathbb{R}^{2}\right)$ are two given vector fields which admit a linear domination, according to Definition 2.3. Let $\psi_{n}(t ; \sigma, w)$ denote the flow of $f_{n}$, and put $\phi_{n}=\psi_{n}(\tau ; \sigma, \cdot)$. Then, as $n \rightarrow$ $+\infty, \phi_{n} \rightarrow \phi_{\infty}$ in $C_{\text {loc }}^{1}\left(\mathbb{R}^{2} ; \mathbb{R}^{2}\right)$.

Lemma 2.9. Let $a, V$ and $f_{*}$ be as in Lemma 2.5. Let $\sigma<\tau$, and put $\phi=$ $\psi(\tau ; \sigma, \cdot)$, where $\psi(t ; \sigma, w)$ denotes the flow of $f_{*}$. Then $\phi \in \mathcal{F}^{*}$, and $\Omega(\phi)=0$.

Proof. Of course, $f_{*}$ is of the same kind as $f_{\infty}$ in the previous lemma, provided we put $p(x, y)=\left(0, V^{\prime}(x)\right)$ and $q(x, y)=\tilde{g}\left(x-x_{0}, y\right)$, where $\tilde{g}$ comes from (6.3). In particular, $\phi$ is $C^{1}$ on $\mathbb{R}^{2}$. Furthermore, according to Remark 2.6, $\phi$ preserves area, so that $\phi \in \mathcal{F}$. On the other hand, $( \pm 1,0)$ are equilibria for $f_{*}$, so that $\phi \in \mathcal{F}^{*}$. Now we divide the proof into three steps.

1) Let us first suppose $a(t) \equiv \lambda$ on $[\sigma, \tau]$, where $\lambda>0$ is a given constant. Then $f_{*}$ turns out to be autonomous on $[\sigma, \tau]$. More precisely, for $y \geq 0, f_{*}$ is nothing but the field $(x, y) \mapsto\left(y, \lambda V^{\prime}(x)\right)$, which admits the invariant curve $\chi(s)=(s, \sqrt{2 \lambda V(s)}), s \in I$. Now Lemma 2.7d ensures that $\Omega(\phi)=0$.

2) $a$ is a positive step function on $[\sigma, \tau]$. Let us put $t_{0}=\sigma$ : then there exist an integer $n \geq 2$ and numbers $t_{k}$ and $\lambda_{k}(k=1, \ldots, n)$ such that $t_{n}=$ $\tau, t_{k-1}<t_{k}(k=1, \ldots, n)$ and $a(t) \equiv \lambda_{k}>0$ on $\left[t_{k-1}, t_{k}[(k=1, \ldots, n)\right.$. Now we can write $\phi=\phi_{n} \circ \cdots \circ \phi_{1}$, where $\phi_{k}=\psi_{k}\left(t_{k} ; t_{k-1}, \cdot\right)$. But $\Omega\left(\phi_{k}\right)=0$, according to the previous step: hence Lemma 2.7b ensures that $\Omega(\phi)=0$.

3) Conclusion. Let $a \in L^{\infty}([\sigma, \tau]), a \geq 0$ : then $a$ can be expressed as the limit, with respect to the $L^{1}$-norm, of a sequence of positive step functions $a_{n}$. Now we can define the fields $f_{n}$ and the corresponding maps $\phi_{n}$ as in Lemma 2.8, provided we put $a_{\infty}=a$, while $p$ and $q$ are as at the beginning of this proof. Then $\phi_{n} \rightarrow \phi_{\infty}=\phi$ in $C_{l o c}^{1}$. But $\Omega\left(\phi_{n}\right)=0$, according to the previous step: hence Lemma 2.7c ensures that $\Omega(\phi)=0$.

Proof of Theorem 2.4. Let $f_{*}(t, u)$ be as in Lemma 2.5: according to the arguments which follow that lemma, we only need to show that (2.4) admits a solution. We point out that $\Gamma=\chi\left(I^{\circ}\right)$, where $\chi(s)=(s, \sqrt{2 l V(s)})$ for any $s \in I$. So, let us define $\psi$ and $\phi$ as in Lemma 2.9, where we put $\sigma=S, \tau=T$. Then $\Omega(\phi)=0$, and we can apply Lemma 2.7 e to $\gamma=\chi$, so as to infer the 
existence of a point $w^{+} \in \phi(\Gamma) \cap \Gamma$. Then the function $u(t)=\psi\left(t ; T, w^{+}\right)$ fulfils (2.4a) and the second condition in (2.4b). Furthermore $w^{+} \in \phi(\Gamma)$, so that there exists a unique $w^{-} \in \Gamma$ such that $w^{+}=\phi\left(w^{-}\right)$. On the other hand, $w^{+}=u(T)=\psi(T ; S, u(S))=\phi(u(S))$, so that $u(S)=w^{-} \in \Gamma$.

It may seem that condition (ii) was not exploited in the proof above: actually, we need it in the proof of Lemma 6.3, which implies Lemma 2.5. On the other hand, as far as we are not interested in monotone solutions, we can directly apply the previous arguments to the field (1.2) rather than to $f_{*}$, so as to skip Lemma 2.5. In this case, however, it is not right, in general, to modify $V$ outside $[-1,1]$ as in Remark 2.2 , so as to get a linear domination on $V^{\prime}$ and be sure that the corresponding flow is defined on the whole phase plane. Then we need to control the behaviour of $V(x)$ for $|x| \geq 1$ as well. Furthermore, in lack of (ii), the condition $V>0$ on ] $-1,1$ [ has to be required explicitly.

Theorem 2.10. Let conditions (i), (iii) and (iv) hold, suppose that $V$ is positive on ] $-1,1\left[\right.$ and $V^{\prime \prime}$ is bounded on $\mathbb{R}$. Then problem $(2.1 a-b)$ admits a solution.

\section{The shooting method}

Let $a$ and $V$ fulfil (i)-(iii). Let us fix positive numbers $r, \mu, \nu$ in such a way that, by virtue of the assumption $V^{\prime \prime}( \pm 1)>0$, the inequalities $\mu \leq V^{\prime \prime}(x) \leq \nu$ hold for $|x \pm 1| \leq r$. Then

$$
\frac{1}{2} \mu(x \pm 1)^{2} \leq V(x) \leq \frac{1}{2} \nu(x \pm 1)^{2}, \quad|x \pm 1| \leq r .
$$

Let $C:=\mathbb{R} \times] 0, r[:$ as we are going to see in the next section, there exists a continuous function $\eta: C \rightarrow \mathbb{R}$ such that the solutions of the following Cauchy problems

$$
\left\{\begin{array}{l}
\ddot{x}(t)=a(t) V^{\prime}(x(t)) \\
x(\tau)=-1+h, \dot{x}(\tau)=\eta(\tau, h)
\end{array} \quad(\tau, h) \in C,\right.
$$

all fulfil the condition $x(-\infty)=-1$. In other words, the points of the kind $(\tau,-1+h, \eta(\tau, h))$ lie in the unstable manifold of the equilibrium $(-1,0)$ of (1.2). Now, let us denote by $U$ the set of all functions $x$ above, and suppose, for the sake of simplicity, that they are defined on the whole real line, according to the final part of Remark 2.2. For any $x \in U$, let us consider the two following cases:

$\left(H^{-}\right)$There exists $\theta \in \mathbb{R}$ such that $\dot{x}(\theta)=0$ and $x(t)<1$ for any $t \leq \theta$.

$\left(H^{+}\right)$There exists $\theta \in \mathbb{R}$ such that $x(\theta)=1$ and $\dot{x}(t)>0$ for any $t \leq \theta$.

Then we denote by $U^{-}$and $U^{+}$the sets of those functions $x \in U$ such that $\left(H^{-}\right)$and $\left(H^{+}\right)$respectively hold, and by $C^{-}$and $C^{+}$the sets of the corresponding pairs $(\tau, h) \in C$ of $(3.2)$.

Remark 3.1. From (3.2) we argue that, whenever $x \in U$, the inequality $\dot{x}>0$ holds definitively to the left: in particular, in the case $\left(H^{-}\right)$, the set $\dot{x}^{-1}(0)$ is closed, non-empty and bounded from below, so that it admits a minimum value $\theta=\theta(x)$, which obviously satisfies $\left(H^{-}\right)$, together with the inequality 
$\dot{x}(t)>0$ for $t<\theta$. On the other hand, if $x \in U^{+}$, the point $\theta=\theta(x)$ of condition $\left(H^{+}\right)$is unique. Finally, if $x \in U$ does not fulfil $\left(H^{-}\right)$neither $\left(H^{+}\right)$, we can put $\theta(x)=+\infty$, so as to define a map $\theta: U \rightarrow]-\infty,+\infty]$.

Remark 3.2. Let us suppose that $x \in U$ does not satisfy $\left(H^{-}\right)$neither $\left(H^{+}\right)$. Then, for any $t \in \mathbb{R}, \dot{x}(t)>0$ and $x(t)<1$, so that, as $t \rightarrow+\infty, x(t)$ must converge to some point of ] - 1,1] at which $V^{\prime}$ vanishes: this point, however, cannot be the point $x_{0}$ of condition (ii), since the inequality $V^{\prime \prime}\left(x_{0}\right)<0$ would imply $\ddot{x}(t)>0$ for $t$ large enough, in contrast with the condition $x(t) \rightarrow x_{0}$ from the left as $t \rightarrow+\infty$. Then the point above is nothing but 1 , that is $x(+\infty)=1$ : since $x(-\infty)=-1$ for any $x \in U,(2.1 \mathrm{~b})$ holds, so that $x$ solves (2.1). In order to find such a function $x$ or, equivalently, a pair $(\tau, h) \in C$ which lies outside $C^{-}$and $C^{+}$, we point out that these two sets are disjoint subsets of the connected, open set ] - 1, 1[ and, as we are going to show in the next result, they are open. Then, under assumption (ii), problem (2.1) surely admits a solution if we are able to show that

$$
C \backslash C^{-} \neq \emptyset, \quad C \backslash C^{+} \neq \emptyset .
$$

Lemma 3.3. $C^{-}$and $C^{+}$are open.

Proof. In order to show that $C^{-}$is open, let us take $\left(\tau_{0}, h_{0}\right) \in C^{-}$and put $\xi_{0}=-1+h_{0}, \eta_{0}=\eta\left(\tau_{0}, h_{0}\right), x(t)=\psi_{1}\left(t ; \tau_{0}, \xi_{0}, \eta_{0}\right)$, where $\psi=\left(\psi_{1}, \psi_{2}\right)$ is the flow of (1.2). According to Remark 3.1, let $\theta_{0}=\theta(x)$ : since $x\left(\theta_{0}\right)>$ $x_{0}$, it is $\ddot{x}\left(\theta_{0}\right)=a\left(\theta_{0}\right) V^{\prime}\left(x\left(\theta_{0}\right)\right)<0$, so that we can find $\theta>\theta_{0}$ such that $\dot{x}(\theta)<0$ and $M(\theta):=\max x\left(\left[\tau_{0}, \theta\right]\right)<1$. Now, let $\varepsilon=\min (-\dot{x}(\theta), 1-M(\theta))$ : from well-known continuity properties of $\psi$, we can find $\delta=\delta(\varepsilon)>0$ such that $\left\|\psi(t ; \tau, \xi, \eta)-\psi\left(t ; \tau_{0}, \xi_{0}, \eta_{0}\right)\right\| \leq \varepsilon$ whenever $\tau_{0} \leq t \leq \theta$, and the values $\left|\tau-\tau_{0}\right|,\left|\xi-\xi_{0}\right|,\left|\eta-\eta_{0}\right|$ do not exceed $\delta$. Furthermore, as we are going to see in Lemma 4.2, the function $\eta$ is continuous: then we can find $\rho_{0}=\rho_{0}(\delta)>0$ such that $\left|\eta(\tau, h)-\eta\left(\tau_{0}, h_{0}\right)\right| \leq \delta$ for $\left|\tau-\tau_{0}\right| \leq \rho_{0},\left|h-h_{0}\right| \leq \rho_{0}$. Now it is easy to see that $(\tau, h) \in C^{-}$for $\left|\tau-\tau_{0}\right| \leq \rho,\left|h-h_{0}\right| \leq \rho$, where $\rho=\min \left(\delta, \rho_{0}\right)$. In a similar way we can prove that $C^{+}$is open.

Remark 3.4. In order to solve problem (2.1) by means of a shooting method we could obviously consider, instead of the unstable manifold of (1.2) in the neighbourhood of $(-1,0)$, its stable manifold near $(1,0)$. Of course, the two approaches are equivalent, and lead to a duality in the assumptions to be done on $a$ and $V$, as is shown by the two alternative conditions (vii) and (viii) of Theorem 5.1. This duality can also be explained by the following, simple argument, which allows to exchange the roles of the two equilibria $(-1,0)$ and $(1,0)$ : let us suppose that, in $(2.1), a$ and $V$ are respectively replaced by $\tilde{a}(t)=a(-t)$ and $\tilde{V}(x)=V(-x)$, and denote by $(\tilde{P})$ the corresponding new problem. Then it is easy to check that, whenever $(\tilde{P})$ admits a solution $\tilde{x}$, the function $x(t)=-\tilde{x}(-t)$ solves the given problem.

Now we are going to deduce some inequalities which follow from the comparison of (2.1a) with an autonomous equation: we introduce them now, even if they will be useful in the following sections. To this end, let $x \in U$ be given: 
we begin to study some properties of the functions

$$
\begin{array}{ll}
E_{\lambda}(t)=\frac{1}{2} \dot{x}(t)^{2}-\lambda V(x(t)), & \lambda>0, t \in \mathbb{R}, \\
E(t)=\frac{1}{2} \dot{x}(t)^{2}-a(t) V(x(t)), & t \in \mathbb{R} .
\end{array}
$$

First of all, we point out that

$$
\dot{E}_{\lambda}(t)=(a(t)-\lambda) V^{\prime}(x(t)) \dot{x}(t) \quad \text { a.e. }
$$

Then, according to Remark 3.1, we put $\theta=\theta(x)$, so that the point $x_{0}$ of condition (ii) is surely attained at some time $\theta_{0}<\theta$. Now, let us suppose $0<l \leq a \leq L$. Hence, by virtue of (3.6):

$$
\begin{array}{ll}
\dot{E}_{l}(t) \geq 0 \geq \dot{E}_{L}(t) & \text { for } t \leq \theta_{0}, \\
\dot{E}_{l}(t) \leq 0 \leq \dot{E}_{L}(t) & \text { for } \quad \theta_{0} \leq t<\theta .
\end{array}
$$

In particular, $[2 L V(x(t))]^{-1 / 2} \dot{x}(t) \leq 1 \leq[2 l V(x(t))]^{-1 / 2} \dot{x}(t)$ for any $t \leq \theta_{0}$, that is to say: $E_{l} \geq 0 \geq E_{L}$ on $\left.]-\infty, \theta_{0}\right]$. Indeed, the last inequalities follow easily from $(3.7,3.8)$, since $E_{l}$ and $E_{L}$ both vanish at $-\infty$. Therefore, whenever $t_{1} \leq t_{2} \leq \theta_{0}, x_{1}=x\left(t_{1}\right), x_{2}=x\left(t_{2}\right)$,

$$
\int_{x_{1}}^{x_{2}}[2 L V(x)]^{-1 / 2} d x \leq t_{2}-t_{1} \leq \int_{x_{1}}^{x_{2}}[2 l V(x)]^{-1 / 2} d x .
$$

Similarly, from the first equality in (3.8) we get $E_{l}(t) \geq E_{l}\left(t_{2}\right) \geq-l V\left(x_{2}\right)$ for all $t \in\left[\theta_{0}, t_{2}\right]$, so as to infer, whenever $\theta_{0} \leq t_{1} \leq t_{2} \leq \theta, x_{1}=x\left(t_{1}\right), x_{2}=x\left(t_{2}\right)$,

$$
t_{2}-t_{1} \leq \int_{x_{1}}^{x_{2}}\left[2 l\left(V(x)-V\left(x_{2}\right)\right)\right]^{-1 / 2} d x
$$

We point out that, in the autonomous case, the function $E(t)$ in (3.5) is nothing but the energy of the system, which keeps constant along any solution. On the contrary, in the general case, $E(t)$ increases or decreases in an opposite way with respect to $a(t)$. Indeed, let $J=\left[t_{0}, t_{1}\right]$ be a given interval, and denote by $\Delta K$ the variation of kinetic energy which $x(t)$ undergoes along the interval $J$. If we multiply both sides of (2.1a) by $\dot{x}(t)$ and integrate over $J$, we get

$$
\Delta K=\frac{1}{2} \dot{x}\left(t_{1}\right)^{2}-\frac{1}{2} \dot{x}\left(t_{0}\right)^{2}=\int_{t_{0}}^{t_{1}} a(t) V^{\prime}(x(t)) \dot{x}(t) d t .
$$

Then, whenever $a \in B V(J)$, integration by parts shows that:

$$
\Delta K=a\left(t_{1}\right) V\left(x\left(t_{1}\right)\right)-a\left(t_{0}\right) V\left(x\left(t_{0}\right)\right)-\int_{t_{0}}^{t_{1}} V(x(t)) d a(t),
$$

that is to say

$$
E\left(t_{1}\right)-E\left(t_{0}\right)=-\int_{t_{0}}^{t_{1}} V(x(t)) d a(t) .
$$

Remark 3.5. The script $a \in L^{\infty}(\mathbb{R})$ could be misleading, when dealing with a Riemann-Stieltjes integral with respect to $d a$, as above. From now on, in order to avoid ambiguities, we agree that the function $a(t)$ is univocally defined 
( not up to negligible sets): of course, this clarification is important when working on those intervals where $a$ is monotone.

Remark 3.6. From (3.13) and the obvious property $E(-\infty)=0$ we argue that, whenever $a(t)$ is monotone on the whole real line, but not constant, it is $E(+\infty) \neq 0$, so that $\dot{x}(+\infty) \neq 0$ as well. Actually, this is the reason why, instead of (2.2), we did not consider the more general case in which $a(t)$ takes two different values $l_{*}$ and $l^{*}$ on the two half-lines $]-\infty, S[$ and $] T,+\infty[$. Indeed, we can easily build a monotone coefficient $a(t)$ such that $a(t) \equiv l_{*}$ for $t<S, a(t) \equiv l^{*}$ for $t>T$ : then, according to the previous argument (2.1) has no solution.

Remark 3.7. Thanks to (3.12), we can easily compare the signs of $\Delta K$ and $\Delta a:=a\left(t_{1}\right)-a\left(t_{0}\right)$ whenever $a$ is monotone on $\left[t_{0}, t_{1}\right]$, and $V$ takes the same value $\beta$ at the points $x\left(t_{0}\right)$ and $x\left(t_{1}\right)$. Indeed, by virtue of (ii), it is $V(x) \geq \beta$ on $\left[x\left(t_{0}\right), x\left(t_{1}\right)\right]$ : then the following relation, which follows at once from (3.12), shows how, in this situation, $\Delta K$ and $\Delta a$ have opposite signs. In particular, this circumstance will be useful in Sect. 5 .

$$
\frac{1}{2} \dot{x}\left(t_{1}\right)^{2}-\frac{1}{2} \dot{x}\left(t_{0}\right)^{2}=-\int_{t_{0}}^{t_{1}}(V(x(t))-\beta) d a(t) .
$$

\section{The unstable manifold}

Throughout this section we suppose that conditions (i)-(iii) of Sect. 2 hold, and that $a(t)$ admits finite, positive limits $l_{*}$ and $l^{*}$ as $t \rightarrow \pm \infty$. More precisely, the following conditions will hold true, where the former is to be explained in the next remark.

(v) $a(t) \downarrow l_{*}$ as $t \rightarrow-\infty, a(t) \downarrow l^{*}$ as $t \rightarrow+\infty$.

(vi) $a(t) \geq \min \left(l_{*}, l^{*}\right)>0$ for any $t \in \mathbb{R}$.

Remark 4.1. By $a(t) \downarrow l_{*}$ we mean that $a(t) \rightarrow l_{*}$ as $t \rightarrow-\infty$ and, for some $S \in \mathbb{R}, a(t)$ is increasing on $]-\infty, S]$. Similarly, $a(t) \downarrow l^{*}$ means that $a(t) \rightarrow l^{*}$ as $t \rightarrow+\infty$ and, for some $T \in \mathbb{R}, a(t)$ is decreasing on $[T,+\infty[$. In both cases we do not require strict monotonicity.

We begin to study the unstable manifold of the field (1.2) in a neighbourhood of $(-1,0)$. To this end, we could exploit general results about the behaviour of a non-autonomous dynamical system near a hyperbolic rest point (see, for instance [9], Thm. IV.3.1). The particular nature of equation (1.1), however, suggests to adopt a variational point of view. To this end, we recall that, whenever $W: \mathbb{R} \rightarrow\left[0,+\infty\left[\right.\right.$ is a $C^{2}$ function such that $W(0)=0$ and $\inf W^{\prime \prime}>0$ then, for any $\tau, h \in \mathbb{R}$, the variational problem

$$
\left.\left.\min \left\{\int_{-\infty}^{\tau}\left(\frac{1}{2} \dot{z}(t)^{2}+a(t) W(z(t))\right) d t ; z \in H^{1}(]-\infty, \tau\right]\right), z(\tau)=h\right\}
$$

has a unique solution $z(t)$, which is characterized by the following properties:

$$
\begin{cases}\ddot{z}(t)=a(t) W^{\prime}(z(t)) & (a) \\ z(\tau)=h, z(-\infty)=0 & (b)\end{cases}
$$


In particular, (4.2a) is the Euler equation of (4.1), while the second condition of (4.2b) is fulfilled by any function $\left.\left.z \in H^{1}(]-\infty, \tau\right]\right)$, as is shown in [3], Cor. VIII.8. In order to fix ideas, let us suppose $h>0$ : then it is easy to see that, for any $t \leq \tau$,

$$
h \geq z(t)>0, \quad \dot{z}(t)>0,
$$

so that the values of $W$ outside $[0, h]$ do not affect problem (4.1). Hence, as far as we are interested in initial values $h \in] 0, r]$, with $r$ as in (3.1), the arguments above hold also for $W(z)=V(-1+z)$. In this case, if we put $\eta(\tau, h)=\dot{z}(\tau)$, it is easy to understand that $\eta$ is the function which appears in (3.2). First of all, we need to know the behaviour of $\eta(\tau, h)$ as $h \rightarrow 0^{+}$. To this end, let us apply the arguments which follow (4.1) to the quadratic approximation of $V(-1+z)$ near 0 , that is $W(z)=\frac{1}{2} \sigma^{2} z^{2}$, where $\sigma=\sqrt{V^{\prime \prime}(-1)}$. We point out that, in this case, the Euler equation of (4.1) is linear:

$$
\ddot{z}=\sigma^{2} a(t) z .
$$

Hence, if we know the solution $z_{1}$ of (4.1) for a fixed pair $\left(\tau_{1}, h_{1}\right)$ it is easy to check that $z(t)=h z_{1}(t) / z_{1}(\tau)$ solves $(4.1)$ for any given pair $(\tau, h)$ : in order to fix ideas, we put $\tau_{1}=0, h_{1}=1$. Then we extend $z_{1}$ to $] 0,+\infty[$ as a solution of (4.4). We also define $z_{2}$ as the solution of (4.4) such that $z_{2}(0)=0, \dot{z}_{2}(0)=1$. From (4.4) and the initial conditions of $z_{1}$ and $z_{2}$, we easily argue that $z_{1}(t)>0$ for $t \in \mathbb{R}$, while $z_{2}(t)<0$ for $t<0$. In particular, for $i=1,2$, the two functions $q_{i}:=\dot{z}_{i} /\left(\sigma z_{i}\right)$ will be respectively considered on $\mathbb{R}($ for $i=1)$ and $]-\infty, 0[$ (for $i=2$ ). Now, let $S \in \mathbb{R}$ be as in Remark 4.1. Then:

$$
\begin{gathered}
E(t):=\frac{1}{2}\left(\dot{z}_{1}(t)^{2}-\sigma^{2} a(t) z_{1}(t)^{2}\right) \leq 0 \text { for } t \leq S, \\
\dot{q}_{1}(t)=\sigma\left(a(t)-q_{1}(t)^{2}\right) \quad \text { a.e. on } \mathbb{R} .
\end{gathered}
$$

Indeed, the function $E(t)$ in (4.5) can be got from (3.5) when replacing $V(x)$ by $W(z)=\frac{1}{2} \sigma^{2} z^{2}$, and $x$ by $z_{1}$. Then, according to the arguments which follow (3.10), $E(t)$ decreases on $]-\infty, S]$ : since $E(-\infty)=0$ (4.5) follows. As regards (4.6), it is enough to recall that $z_{1}$ solves (4.4). Now, let $0<\mu<\sigma \sqrt{l_{*}}, b \in$ $L^{\infty}(\mathbb{R}), \beta:=b(-\infty) \in \mathbb{R}$ and put, for $\left.\left.t \leq 0, J_{1}(t)=\right]-\infty, t\right], J_{2}(t)=[t, 0]$. Then, as $t \rightarrow-\infty$ :

$$
\begin{gathered}
(a) q_{1}(t) \rightarrow \sqrt{l_{*}}, \quad(b) q_{2}(t) \rightarrow-\sqrt{l_{*}}, \\
(a) z_{1}(t) e^{-\mu t} \rightarrow 0, \quad(b) z_{2}(t) \rightarrow-\infty, \quad(c) z_{1}(t) z_{2}(t) \rightarrow \frac{-1}{2 \sigma \sqrt{l_{*}}}, \\
p_{i}(t):=z_{i}(t)^{-1} \int_{J_{i}(t)} b(s) z_{i}(s) d s \rightarrow \frac{\beta}{\sigma \sqrt{l_{*}}}, \quad i=1,2 .
\end{gathered}
$$

Indeed, the function $E(t)$ of (4.5) and the right-hand side of (4.6) have opposite signs, so that $q_{1}(t)$ is increasing for $t \leq S$. Since $q_{1}(t) \geq 0$ [because (4.3) holds for $z=z_{1}$ ], it converges from above to some non-negative, finite value as $t \rightarrow-\infty$ : actually, from (4.6) we argue that this value is nothing but $\sqrt{l_{*}}$, so as to get (4.7a). As regards (4.8a), let us take $\lambda \in] \mu / \sigma, \sqrt{l_{*}}[$ : thanks to (4.7a) and the definition of $q_{1}$, we find $\tau \leq 0$ such that $\dot{z}_{1}(s) / z_{1}(s) \geq \mu_{1}$ for 
$s \leq \tau$, where $\mu_{1}:=\lambda \sigma>\mu$. Now, for any $t \leq \tau$, we can integrate the previous inequality from $t$ to $\tau$ and put $K=z_{1}(\tau) \exp \left(-\mu_{1} \tau\right)$, so as to get the inequalities $0 \leq z_{1}(t) \leq K \exp \left(\mu_{1} t\right)$, which imply (4.8a). On the other hand (4.8b) follows easily from the initial conditions of $z_{2}$, since it solves (4.4). As regards (4.8c), we remark that the Wronsk determinant $w(t)$ of $z_{1}$ and $z_{2}$ is constant, and $w(0)=1$. Now we can regard the identity $w \equiv 1$ as a differential equation with respect to $z_{2}$ : since $z_{2}(0)=0$, we get $z_{2}(t):=z_{1}(t) \int_{0}^{t} z_{1}(s)^{-2} d s$, so that $z_{1}(t) z_{2}(t)$ can be seen as the ratio between the two functions $\int_{0}^{t} z_{1}(s)^{-2} d s$ and $z_{1}(t)^{-2}$, which respectively diverge to $-\infty$ and $+\infty$ as $t \rightarrow-\infty$. Now a simple application of de l'Hopital's rule, together with (4.7a), yields (4.8c). In order to get $(4.7 \mathrm{~b})$, we remark that $w=\sigma z_{1} z_{2}\left(q_{2}-q_{1}\right)$ : since $w \equiv 1$, (4.7b) follows from the previous relation, (4.7a) and (4.8c). Finally, in (4.9), we point out that $p_{1}$ is well-defined, since (4.8a) ensures that $z_{1}$ is summable on ] $\left.-\infty, t\right]$. Furthermore, for $i=1,2, p_{i}(t)$ is the ratio between the two functions $\int_{J_{i}(t)} b(s) z_{i}(s) d s$ and $z_{i}(t)$. Now, for $i=1$, they both converge to 0 , thanks also to (4.8). On the other hand, for $i=2$, and provided that $\beta \neq 0$, they surely diverge to $\pm \infty$ and $-\infty$ respectively, where the first limit depends on the sign of $\beta$ : in both cases, we can apply again de l'Hopital's rule, and get (4.9) by virtue of (4.7). Also the case $i=2, \beta=0$, which was left apart, can be easily tackled: indeed, according to the previous argument, the ratio $\Phi(t)$ between $\int_{J_{2}(t)} z_{2}(s) d s$ and $z_{2}(t)$ converges to $1 / \sigma \sqrt{l_{*}}$, and $\left|p_{2}(t)\right| \leq|b(t)| \Phi(t)$, where, in order to justify the last inequality, it is useful to recall that $z_{2}(t)<0$ for $t<0$, so that $-\left|z_{2}\right|$ can replace $z_{2}$ in the definition of $\Phi$. Then $p_{2}(t) \rightarrow 0$, as required by (4.9) in this particular case.

Now we suppose $\beta=0$, denote by $(\mathcal{L})$ the complete linear equation $\ddot{z}=$ $\sigma^{2} a z+b$ and try to solve the following problem: for any $\tau, h \in \mathbb{R}$ find $\eta \in \mathbb{R}$ in such a way that the solution of $(\mathcal{L})$ which fulfils the initial data $z(\tau)=$ $h, \dot{z}(\tau)=\eta$ vanishes at $-\infty$. To this end we put

$$
\begin{aligned}
& p(t)=z_{2}(t) \int_{-\infty}^{t} b(s) z_{1}(s) d s+z_{1}(t) \int_{t}^{0} b(s) z_{2}(s) d s, \\
& z\left(t ; c_{1}, c_{2}\right)=c_{1} z_{1}(t)+c_{2} z_{2}(t)+p(t), \quad t, c_{1}, c_{2} \in \mathbb{R} .
\end{aligned}
$$

For $t \leq 0$ it is $p(t)=z_{1}(t) z_{2}(t)\left(p_{1}(t)+p_{2}(t)\right)$, as we can easily argue from the definition of $p_{1}$ and $p_{2}$ in (4.9). Then, according to (4.9) and (4.8c), we get $p(-\infty)=0$, since $\beta=0$. On the other hand, $p$ solves $(\mathcal{L})$, as we can see through variation of constants: hence any solution $z$ of $(\mathcal{L})$ takes the form (4.11), and $z(-\infty)=0$ if and only if $c_{2}=0$, according to the behaviour of $z_{1}, z_{2}$ and $p$ as $t \rightarrow-\infty$. Then the required value of $\eta$ is given by $c_{1} \dot{z}_{1}(\tau)+\dot{p}(\tau)$, where $c_{1} z_{1}(\tau)+p(\tau)=h$. If $w_{1}$ denotes the Wronsk determinant of $z_{1}$ and $p$, we actually get $\eta z_{1}(\tau)=h \dot{z}_{1}(\tau)+w_{1}(\tau)$. Now, since $z_{1}$ and $p$ both vanish at $-\infty$, it is easy to infer the same property on $\dot{z}_{1}$ and $\dot{p}$, so that $w_{1}(-\infty)=0$. Furthermore, $\dot{w}_{1}=b z_{1}$, because $z_{1}$ and $p$ respectively solve $(4.4)$ and $(\mathcal{L})$. Then $w_{1}(\tau)=\int_{-\infty}^{\tau} b(s) z_{1}(s) d s$, so that:

$$
\eta=\frac{1}{z_{1}(\tau)}\left(h \dot{z}_{1}(\tau)+\int_{-\infty}^{\tau} b(s) z_{1}(s) d s\right) .
$$


Lemma 4.2. Let $\eta(\tau, h)$ be as in (3.2), $S \in \mathbb{R}$ as in Remark 4.1. Then:

(a) $\eta(\tau, h) / h \rightarrow \sigma q_{1}(\tau)$ as $h \rightarrow 0^{+}$, uniformly with respect to $\tau \leq S$.

(b) The function $(\tau, h) \mapsto \eta(\tau, h)$ is continuous.

(c) $q_{1}(\tau) \geq \sqrt{m}$ whenever, for some $m>0$, it is $a(t) \geq m$ on $\left.]-\infty, \tau\right]$. If the latter inequality is strict on a set of positive measure, the former is strict as well.

Proof. (a) Let $\tau \leq S, h \in] 0, r]$ be given, $z(t)=u(t ; \tau, h)$ be the corresponding solution of (4.1), where $W(z)=V(-1+z)$, so that $\eta(\tau, h)=\dot{z}(\tau)$. Let us put $\gamma(z)=V^{\prime}(-1+z)-\sigma^{2} z$, consider the function $\beta(t ; \tau, h)=$ $a(t) \gamma(u(t ; \tau, h))$ and denote by $B(\tau, h)$ its norm in $\left.\left.L^{\infty}(]-\infty, \tau\right]\right)$. Let also $\Omega(h)$ and $\omega(h)$ stand for the maximum values on $[0, h]$ of $|\gamma|$ and $\left|\gamma^{\prime}\right|$, so that $\Omega(h) \leq h \omega(h)$ and, according to $(4.3), B(\tau, h) \leq L \Omega(h)$, where $L=\|a\|_{\infty}$. Since $\gamma^{\prime}(0)=0$, we also get $\omega(h) \rightarrow 0$ as $h \rightarrow 0^{+}$. Furthermore, the ratio between $\int_{-\infty}^{\tau} z_{1}(s) d s$ and $z_{1}(\tau)$ admits the finite limit $\left(\sigma \sqrt{l_{*}}\right)^{-1}$ as $\tau \rightarrow-\infty$, as we can argue when putting $i=1, b \equiv \beta=1$ and $t=\tau$ in (4.9): then it takes a maximum value $\Phi$ on $]-\infty, S]$. On the other hand, $z$ solves the equation $\ddot{z}=\sigma^{2} a(t) z+b(t)$, where $b=\beta(\cdot ; \tau, h)$ : in particular, it is $b(-\infty)=0$, since $\gamma(0)=0$. Hence the value $\eta$ we get from (4.12) by putting in it $b=\beta(\cdot ; \tau, h)$ is nothing but $\eta(\tau, h)=\dot{z}(\tau)$. Thanks to the previous arguments, we get the following evaluation, which proves our claim:

$$
\left|\frac{\eta(\tau, h)}{h}-\sigma q_{1}(\tau)\right| \leq \frac{B(\tau, h)}{h z_{1}(\tau)} \int_{-\infty}^{\tau} z_{1}(s) d s \leq \frac{L \Omega(h)}{h} \Phi \leq L \Phi \omega(h) .
$$

(b) From (4.13) we argue that $\eta$ is locally bounded: then we only need to prove that its graph is closed. To this end, let us put $w(t ; \tau, h, \eta)=$ $\psi_{1}(t ; \tau,-1+h, \eta)$, where $\psi=\left(\psi_{1}, \psi_{2}\right)$ stands for the flow of (1.2). Then $u(t ; \tau, h)=w(t ; \tau, h, \eta(\tau, h))$ and, according to (4.12), the value $\eta=\eta(\tau, h)$ can be characterized as the only solution to the equation

$$
\eta z_{1}(\tau)-h \dot{z}_{1}(\tau)-j(\tau, h, \eta)=0,
$$

where we put $j(\tau, h, \eta)=\int_{-\infty}^{\tau} a(t) \gamma(w(t ; \tau, h, \eta)) z_{1}(t) d t$. We recall that, according to (2.3), we can suppose that $u$ (and therefore $w$ ) is defined everywhere, as a function of $t$, and also that $\gamma$ is bounded. Furthermore, according to $\left.\left.(4.8 \mathrm{a}), z_{1} \in L^{1}(]-\infty, \tau\right]\right)$. Then $j$ is well defined, and it is easy to prove that it is continuous: indeed, whenever $\left(t_{i}, h_{i}, \eta_{i}\right) \rightarrow(t, h, \eta)$ as $i \rightarrow+\infty$, the Lebesgue theorem, together with the continuity of $\gamma$ and $w$, ensures the expected convergence. Hence (4.14) defines a closed set of points $(\tau, h, \eta)$, as required.

(c) Let $\tau$ be fixed, and put in (4.1) $W(z)=\frac{1}{2} \sigma^{2} z^{2}, h=z_{1}(\tau)$ : then $z_{1}$ obviously minimizes the corresponding functional $\Phi_{\tau}(\cdot ; a)$. Since $z_{1}$ solves (4.4), a simple integration by parts on the first term of $m_{1}:=\Phi_{\tau}\left(z_{1} ; a\right)$ shows that $m_{1}=\frac{1}{2} z_{1}(\tau) \dot{z}_{1}(\tau)$. Now, let us replace in the previous problem $a(t)$ by the constant $m$ : then the corresponding solution is $z^{*}(t)=$ $z_{1}(\tau) \exp (\lambda(t-\tau))$, where $\lambda=\sigma \sqrt{m}$, so that $m^{*}:=\Phi_{\tau}\left(z^{*} ; m\right)$ turns out to be $\frac{1}{2} \lambda z_{1}(\tau)^{2}$. In order to get our first claim, according to the definition 
of $q_{1}$ and the previous evaluations, it is enough to prove the inequality $m_{1} \geq m^{*}$, which follows from $m_{1} \geq \Phi_{\tau}\left(z^{*} ; a\right) \geq m^{*}$. As regards the second assertion, we point out that $z^{*}>0$, so that, in this case, the strict inequality $\Phi_{\tau}\left(z^{*} ; a\right)>m^{*}$ holds.

The proof of the two conditions which appear in (3.3) will be given in Sect. 5, and requires, respectively, evaluations from below and above of the following ratio, which are achieved in the next result:

$$
R(\tau, h)=\frac{1}{2} \frac{\eta(\tau, h)^{2}}{V(-1+h)}, \quad(\tau, h) \in C .
$$

Lemma 4.3. Let $S$ be as in Remark 4.1, $R(\tau, h)$ as in (4.15). Then:

(a) For any $l_{1}>l_{*}$ there exists $\left.h_{1} \in\right] 0, r\left[\right.$ such that $R(\tau, h) \leq l_{1}$ whenever $\tau \leq S, 0<h \leq h_{1}$.

(b) Let $l_{*} \geq l^{*}:$ for any $T \in \mathbb{R}$ there exists a pair $(\tau, h) \in C$ such that $\tau \geq T$ and $R(\tau, h) \geq a(\tau)$.

Proof. (a) Since $2 h^{-2} V(-1+h) \rightarrow V^{\prime \prime}(-1)=\sigma^{2}$ as $h \rightarrow 0^{+}$, Lemma $4.2 \mathrm{a}$ entails that, as $h \rightarrow 0^{+}$, the following relation holds uniformly with respect to $\tau \leq S$, so that our claim follows from (4.7a).

$$
R(\tau, h)=\frac{\eta(\tau, h)^{2}}{h^{2}} \frac{h^{2}}{2 V(-1+h)} \rightarrow q_{1}(\tau)^{2} .
$$

(b) If $l_{*}>l^{*}$, it is obviously $a(t)>l^{*}$ on a set of positive measure $\Delta$, but also if $l_{*}=l^{*}$, provided that $a(t)$ is not constant: on the other hand, when $a(t) \equiv l_{*}=l^{*}$, the inequality to be proved becomes a trivial identity. So, let $\Delta$ be as above, and fix $T \in \mathbb{R}$ : of course, we can suppose that $\Delta \cap]-\infty, T]$ has positive measure. Then, according to the last claim of Lemma $4.2 \mathrm{c}$ (where we put $m=l^{*}$ ), $q_{1}(t)>\sqrt{l^{*}}$ for $t \geq T$. Thanks to (4.16), we only need to show that the inequality $q_{1}(\tau)^{2}>a(\tau)$ holds for some $\tau \geq T$. Let us suppose, by contradiction, that $q_{1}(t) \leq \sqrt{a(t)}$ on $\left[T,+\infty\left[\right.\right.$. Then, from (4.6), we should argue $\dot{q}_{1} \geq 0$ on that half-line, so that $q_{1}$ should converge from below to a limit which, according to (4.6), is nothing but $\sqrt{l^{*}}$. Hence $q_{1}(t) \leq \sqrt{l^{*}}$ for $t \geq T$, in contrast with the strict inequality we got before.

\section{The asymptotically autonomous case}

In order to introduce the main result of this section, we put forward the following conditions on the speed at which $a(t)$ approaches its limits as $t \rightarrow \pm \infty$, where $c$ is a positive constant to be specified below:

$$
\begin{aligned}
e^{-2 c t}\left(a(t)-l_{*}\right) & \rightarrow+\infty \quad \text { as } t \rightarrow-\infty \\
e^{2 c t}\left(a(t)-l^{*}\right) & \rightarrow+\infty \quad \text { as } t \rightarrow+\infty .
\end{aligned}
$$

Theorem 5.1. Let conditions (i)-(iii) of Sect. 2 and (v), (vi) of Sect. 4 hold, and put $c^{-}=\sqrt{l^{*} V^{\prime \prime}(-1)}, c^{+}=\sqrt{l_{*} V^{\prime \prime}(1)}$. Then problem $(2.1)$ admits a solution, provided that one of the two following assumptions is satisfied: 
(vii) $l_{*} \geq l^{*}$, and (5.1) holds for some $c<c^{-}$.

(viii) $l_{*} \leq l^{*}$, and (5.2) holds for some $c<c^{+}$.

First of all we are going to get some evaluations on the transition times of the trajectories of (3.2). To this end, we exploit inequalities $(3.9,3.10)$, where we put $l=\min \left(l_{*}, l^{*}\right), L=\|a\|_{\infty}$. In particular, we are interested in the time that a given function $x \in U$ needs for connecting two points at which $V$ takes the same value. To this end, we recall that the restrictions of $V$ to the two intervals $\left[-1, x_{0}\right]$ and $\left[x_{0}, 1\right]$ are both strictly monotone: then, for any $\left.h \in] 0,1+x_{0}\right]$, there exists a unique $\left.\left.\delta(h) \in\right] 0,1-x_{0}\right]$ such that

$$
V(1-\delta(h))=V(-1+h) \text {. }
$$

For any $(\tau, h) \in C$ let us consider the corresponding solution $x$ of (3.2): then the value $\theta=\theta(x)$ of Remark 3.1 can be written as $\theta=\theta(\tau, h)$, and we can put $\xi(\tau, h)=x(\theta(\tau, h))$, even in the case $\theta(\tau, h)=+\infty$. Now, for any $(\tau, h) \in C$ such that $\xi(\tau, h)>1-\delta(h)$, let $T(\tau, h)$ be the time that the solution $x$ of $(3.2)$ needs for connecting the points $-1+h$ and $1-\delta(h)$. Furthermore, let us put, for any $\lambda>0, h \in] 0, r[$,

$$
\begin{aligned}
I_{*}(\lambda, h) & =\int_{-1+h}^{x_{0}}[2 \lambda V(x)]^{-1 / 2} d x, \\
I^{*}(\lambda, h) & =\int_{x_{0}}^{1-\delta(h)}[2 \lambda(V(x)-V(1-\delta(h)))]^{-1 / 2} d x,
\end{aligned}
$$

and denote by $t_{1}, \theta_{0}$ and $t_{2}$ the times at which, respectively, $x$ takes the values $-1+h, x_{0}, 1-\delta(h)$. Then

$$
\begin{gathered}
I_{*}(L, h) \leq \theta_{0}-t_{1} \leq I_{*}(l, h), \\
t_{2}-\theta_{0} \leq I^{*}(l, h) .
\end{gathered}
$$

Indeed (5.4) follows from (3.9), where $x_{1}=-1+h, x_{2}=x_{0}$ (5.5) from (3.10), where $x_{1}=\theta_{0}, x_{2}=1-\delta(h)$. But $T(\tau, h)=t_{2}-t_{1}$, so that, if we put $I(l, h)=I_{*}(l, h)+I^{*}(l, h)$,

$$
I_{*}(L, h) \leq T(\tau, h) \leq I(l, h) .
$$

Remark 5.2. When $x \in U^{+}$, the right hand side of (3.10) can be replaced by $\int_{x_{1}}^{x_{2}}(2 l V(x))^{-1 / 2} d x$, since $E_{l}(t) \geq E_{l}(\theta) \geq 0$, where $\theta$ is taken from $\left(H^{+}\right)$. Then the first inequality in (3.1), where we take the minus sign, implies that, whenever $1-r \leq x_{1} \leq x_{2}<1$ :

$$
t_{2}-t_{1} \leq \frac{1}{\sqrt{\mu l}} \log \frac{1-x_{1}}{1-x_{2}} .
$$

In the two following results we are going to prove that both conditions in (3.3) take place, so that Theorem 5.1 will follow easily. We recall that $V$ fulfils conditions (i) and (ii) of Sect. 2: in particular, in Lemma 5.3, condition (ii) is to ensure the evaluation from above in (5.6). From now on, in order to fix ideas, we suppose $l_{*} \geq l^{*}$, so that $l=l^{*}$.

Lemma 5.3. Let conditions (i)-(iii), (v), (vi) hold, and $l_{*} \geq l^{*}$. Let (5.1) be satisfied by some $c \in] 0, c^{-[}$: then $C \backslash C^{+} \neq \emptyset$. 
Proof. Let us argue by contradiction, and suppose that $\left(H^{+}\right)$holds for any $x \in U$ : then we are going to build a function $x \in U^{-}$. First of all, we suppose $c \leq \sqrt{l^{*} \mu}$, as we can actually do thanks to a suitable choice of the number $r$ in (3.1). Then we take $S$ as in Remark 4.1 and, by virtue of (5.1), we choose $t_{1}<S$ such that

$$
e^{-2 c\left(S-t_{1}\right)}<\frac{\mu}{\nu} \frac{a\left(t_{1}\right)-l_{*}}{a\left(t_{1}\right)} .
$$

Now we can find $\rho \in] 0,1\left[\right.$ such that $\rho^{2}$ is strictly included between the two sides of the inequality above, so as to get

$$
\begin{gathered}
l_{1}:=a\left(t_{1}\right)\left(1-\frac{\nu}{\mu} \rho^{2}\right)>l_{*}, \\
S-t_{1} \geq \frac{1}{c} \log \frac{1}{\rho} .
\end{gathered}
$$

Since $l_{1}>l_{*}$, we can find $h_{1}>0$ as in Lemma 4.3 a and take $\left.h \in\right] 0, h_{1}[$ such that $\delta(h) \leq r$ and $R(\tau, h) \leq l_{1}$ for any $\tau \leq S$. We can also suppose that $I_{*}(L, h)>t_{1}-S$, since $I_{*}(L, h) \rightarrow+\infty$ as $h \rightarrow 0^{+}$, and take $\tau_{0}$ such that $t_{1}-\tau_{0}>I(l, h)$. Then, from (5.6),

$$
\tau_{0}+T\left(\tau_{0}, h\right)<t_{1}<S+T(S, h),
$$

where the function $T(\tau, h)$ was defined after (5.3), and can be easily shown to be continuous on its domain, which contains $C^{+}$. Then we can find $\left.\tau \in\right] \tau_{0}, S[$ such that $\tau+T(\tau, h)=t_{1}$ : we claim that $(\tau, h) \in C^{-}$. More precisely, as we are going to show, the function $x \in U$ which corresponds to the previous choice of $\tau$ and $h$ fulfils $\left(H^{-}\right)$at the first time $\theta$ at which $x(\theta)=1-\rho \delta(h)$. First of all, we need to show that $\theta \leq S$. To this end, let us apply (5.7) with $x_{1}=x\left(t_{1}\right)=1-\delta(h), t_{2}=\theta, x_{2}=x(\theta)=1-\rho \delta(h)$, so as to infer that $\theta-t_{1}$ does not exceed $(l \mu)^{-1 / 2} \log (1 / \rho)$. Then it is enough to recall that $c \leq \sqrt{l^{*} \mu}, l=l^{*}$ and (5.10) holds in order to get $\theta \leq S$. In particular, we can suppose that $a(t)$ is increasing for $t \leq \theta$. Moreover:

$$
\begin{gathered}
\frac{1}{2} \dot{x}\left(t_{1}\right)^{2}-\frac{1}{2} \eta(\tau, h)^{2} \leq 0, \\
V(x(\theta))=V(1-\rho \delta(h)) \leq \frac{1}{2} \nu \rho^{2} \delta(h)^{2} \leq \frac{\nu}{\mu} \rho^{2} V(1-\delta(h)) .
\end{gathered}
$$

Indeed (5.12) can be deduced from (3.1), while (5.11) follows from (3.14), where we put $t_{0}=\tau$ and recall that $\dot{x}(\tau)=\eta(\tau, h)$ and $V$ takes the same value on $x(\tau)=-1+h$ and $x\left(t_{1}\right)=1-\delta(h)$. Then

$$
\begin{aligned}
\frac{1}{2} \dot{x}(\theta)^{2} & =\frac{1}{2} \dot{x}\left(t_{1}\right)^{2}+\int_{t_{1}}^{\theta} a(t) V^{\prime}(x(t)) \dot{x}(t) d t \\
& \leq \frac{1}{2} \eta(\tau, h)^{2}+a\left(t_{1}\right)(V(x(\theta))-V(1-\delta(h)))
\end{aligned}
$$




$$
\begin{aligned}
& \leq l_{1} V(-1+h)+a\left(t_{1}\right)\left(\frac{\nu}{\mu} \rho^{2}-1\right) V(1-\delta(h)) \\
& =V(-1+h)\left[l_{1}-a\left(t_{1}\right)\left(1-\frac{\nu}{\mu} \rho^{2}\right)\right]=0 .
\end{aligned}
$$

In particular: the first equality follows from (3.11), in which we put $t_{0}=$ $t_{1}, t_{1}=\theta$. The first inequality holds by virtue of (5.11) and also because $a(t)$ is increasing on ] $-\infty, S]$, while $V^{\prime}(x(t))<0$ for $t \geq t_{1}>\theta_{0}$. The second one, on the contrary, follows from the inequality $R(\tau, h) \leq l_{1}$ and (5.12). Finally, the two last equalities follow from (5.3) and the definition of $l_{1}$, which is given in (5.9). Now, from the previous chain of relations, we get $\dot{x}(\theta)=0$ : but $\theta$ is the first time at which $x(\theta)=1-\rho \delta(h)<1$, so that $x \in U^{-}$, a contradiction.

Lemma 5.4. Let conditions (i)-(iii), (v), (vi) hold, and suppose that $l_{*} \geq l^{*}$. Then $C \backslash C^{-} \neq \emptyset$.

Proof. Let $T \in \mathbb{R}$ be as in Remark 4.1, take $(\tau, h)$ as in Lemma $4.3 \mathrm{~b}$ and consider the corresponding function $x$ of (3.2). Let us suppose, by contradiction, that $x \in U^{-}$, and take $\theta$ as in $\left(H^{-}\right)$. Since $\dot{x}(\theta)=0$ and (3.13) applies to $t_{0}=\tau$ and $t_{1}=\theta$, the following equalities hold true:

$$
-a(\theta) V(x(\theta))=E(\theta)=E(\tau)-\int_{\tau}^{\theta} V(x(t)) d a(t) .
$$

We recall that $E(\tau)=\frac{1}{2} \eta(\tau, h)^{2}-a(\tau) V(-1+h)$, and $R(\tau, h) \geq a(\tau)$, so that $E(\tau) \geq 0$. Since the the integral above is non-positive, from the second equality of $(5.13)$ we get $E(\theta) \geq 0$, while the first one entails $E(\theta)<0$ : this contradiction shows that, actually, $x \notin U^{-}$, so that $C \backslash C^{-} \neq \emptyset$.

Proof of Theorem 5.1. As we already explained, it is enough to prove (3.3). Furthermore, thanks to Remark 3.4, we only need to consider the case in which (vii) holds: now our claim follows from Lemmas 5.3-4.

Remark 5.5. In lack of condition (vii) or (viii), Theorem 5.1 does not work, as is shown by the simple example of Remark 3.6. Nevertheless, we think it interesting to exhibit also examples in which $a$ is not monotone. To this end, let $l_{*}, l^{*}>0, S, T \in \mathbb{R}, a \in L^{\infty}(\mathbb{R})$ be such that $l^{*} \neq l_{*}, S<T, a \geq 0, a(t) \equiv l_{*}$ for $t<S$ and $a(t) \equiv l^{*}$ for $t>T$. We are going to show that (2.1) has no solutions if the difference $\Delta:=T-S$ is too small with respect to the norm $R$ of $a$ in $L^{\infty}([S, T])$. Indeed, let us suppose that $(2.1)$ admits a solution $x$ and put, for any $t \in[S, T], \rho(t)=\|u(t)-u(S)\|$, where $u=(x, \dot{x})$. Let $M_{0}$ and $M_{1}$ denote the respective norms of the functions $\frac{1}{2} V^{\prime} V^{-1 / 2}$ and $V^{\prime \prime}$ in $L^{\infty}(]-1,1[)$ : on this subject, we point out that $\frac{1}{2} V^{\prime} V^{-1 / 2}$ is actually bounded on ] $-1,1\left[\right.$, since its square admits the finite limits $\frac{1}{2} V^{\prime \prime}( \pm 1)$ as $x \rightarrow \pm 1$. We also put $\sigma=\sqrt{l^{*} / l_{*}}$, and denote by $K$ and $\gamma$ suitable positive constants. Then: 


$$
\begin{aligned}
& \text { (a) } \dot{x}(S)=\sqrt{2 l_{*} V(x(S))}, \quad \text { (b) } \dot{x}(T)=\sqrt{2 l^{*} V(x(T))}, \\
& |\dot{x}(T)-\sigma \dot{x}(S)|=\sqrt{2 l^{*}}|\sqrt{V(x(T))}-\sqrt{V(x(S))}| \\
& \leq \sqrt{2 l^{*}} M_{0}|x(T)-x(S)|, \\
& \dot{x}(S)|1-\sigma| \leq|\dot{x}(T)-\sigma \dot{x}(S)|+|\dot{x}(T)-\dot{x}(S)| \\
& \leq K\|u(T)-u(S)\|=K \rho(T), \\
& \|\dot{u}(S)\| \leq|\dot{x}(S)|+|\ddot{x}(S)| \leq|\dot{x}(S)|+R \mid V^{\prime}(x(S) \mid \leq \gamma \dot{x}(S) .
\end{aligned}
$$

Of course, (5.14) is due to the conditions $x( \pm \infty)= \pm 1$, and entails the equality in (5.15). Since $M_{0}$ is a Lipschitz constant for $\sqrt{V}$, the inequality in (5.15) holds as well, and implies, in turn, the last inequality of (5.16), provided we take $K=\sqrt{2 l^{*} M_{0}^{2}+1}$. Finally, as regards the last inequality in (5.17), we point out that $\left|V^{\prime}\right|$ does not exceed $2 M_{0} \sqrt{V}$ (in particular at $x=x(S)$ ), and recall (5.14a): then it is enough to put $\gamma=R M_{0} \sqrt{2 / l_{*}}+1$. We also recall, in (5.16) and (5.17), that $\dot{x}(S)>0$. Now, let $f$ be as in (1.2), $L=R M_{1}+1$. We remark that the vector function $u$ is absolutely continuous, so that $\rho$ enjoys the same property. Then:

$$
\begin{array}{rlrl}
|\dot{\rho}(t)| & \leq\|\dot{u}(t)\|=\|f(t, u(t))\| & \\
& \leq\|f(t, u(S))\|+\|f(t, u(t))-f(t, u(S))\| & \\
& \leq\|\dot{u}(S)\|+L\|u(t)-u(S)\| \leq \gamma \dot{x}(S)+L \rho(t) & & \text { a.e. on }[S, T], \\
& \rho(t) \leq(\gamma / L) \dot{x}(S)(\exp (L(t-S))-1), & t \in[S, T] .
\end{array}
$$

Indeed, the field $f(t, \cdot)$ obviously admits the Lipschitz constant $L$, so that the third inequality (from the beginning) in (5.18) holds true, while the last one follows from (5.17). Finally, from (5.18), Gronwall's Lemma and the condition $\rho(S)=0$, we easily argue (5.19). Now, let us put $t=T$ in (5.19), and compare the resulting inequality with (5.16): after removing the common factor $\dot{x}(S)>0$, we find that $|1-\sigma|$ does not exceed $C\left(e^{L \Delta}-1\right)$, where $C=\gamma K / L$. Hence:

$$
\Delta \geq \frac{1}{L} \log \left(1+\frac{|1-\sigma|}{C}\right) .
$$

We point out that the constants $L$ and $C$ do not depend on the solution $x$, while they depend on $a$ only through the values $R, l_{*}, l^{*}$. In particular, if we suppose that $l_{*}$ and $l^{*}$ are given, we can write the right-hand side of (5.20) as $\lambda(R)$, and recall that $\lambda(R)>0$, since $l^{*} \neq l_{*}$ entails $\sigma \neq 1$. Now, in order to build the required counter-example, we only need to fix $R>0$, and take $S, T \in \mathbb{R}$ in such a way that $0<\Delta=T-S<\lambda(R)$ : then, according to (5.20), (2.1) cannot admit solutions, as soon as $0 \leq a \leq R$ on $[S, T]$.

\section{Acknowledgements}

The author thanks Luís Sanchez for the useful discussions on the subject of this work. He thanks the referee as well, for having noticed a mistake in an example of the original version, so as to allow the author to get the new results of Sect. 2, and also for suggesting the more general setting of the present Remark 5.5. 


\section{Appendix}

In this section we are going to prove Lemma 2.5. To this end, we put forward the following Lemma 6.1, where the origin plays the role of the point $x_{0}$ of condition (ii). In a similar way, in Lemma 6.3, the variable $x$ is shifted, with respect to Lemma 2.5, in order to bring the point $x_{0}$ to the origin.

Lemma 6.1. Let $I=[\sigma, \tau] \subseteq \mathbb{R}, \Omega \subseteq \mathbb{R}^{2}, g(t, u) \in \mathbb{R}^{2}$ be a vector field on $I \times \Omega$ which is measurable with respect to $t$ and continuous with respect to $u$. For any $t \in I, u \in \Omega, \lambda \in \mathbb{R}$ let us define $Q_{\lambda}(t, u)$ as $g(t, u) \cdot(u+\lambda J u)$, where $J:(x, y) \mapsto(y,-x)$. Let $u=(x, y) \in A C(I ; \Omega), \dot{u}(t)=g(t, u(t))$ a.e. on $I, \rho(t):=\|u(t)\|>0$ on $I$. Let $\theta \in A C(I ; \mathbb{R})$ be such that $x+i y=\rho e^{i \theta}$ on $I$, and put $\Delta \theta=\theta(\tau)-\theta(\sigma)$. Suppose that, for some $\lambda \geq 0, Q_{\lambda} \geq 0$ on $I \times \Omega$. Then $\rho(\tau) \geq \rho(\sigma) \exp (\lambda \Delta \theta)$.

Proof. It is known that a function $\theta$ as above exists, and $\Delta \theta$ does not depend on its choice. Furthermore $\dot{\theta}(t) \rho(t)^{2}=-\dot{u}(t) \cdot J u(t), \rho(t) \dot{\rho}(t)=u(t) \cdot \dot{u}(t)$ : since $\dot{u}(t)=g(t, u(t))$, from $Q_{\lambda}(t, u(t)) \geq 0$ we get the inequality $\lambda \dot{\theta} \leq \dot{\rho} / \rho$, and we only need to integrate it on $I$.

Definition 6.2. Let $g(t, u)$ be a vector field which is measurable with respect to $t$ and lipschitzian with respect to $u, \psi(t ; \tau, u)$ its flow, $C$ a closed set, $u \in$ $\partial C$ : we say that $u$ is an exit point for $C$ with respect to $g$, and write $u \in$ $\Gamma^{+}(C)$, if there exists $\tau \in \mathbb{R}$ such that $\psi([\tau, \sigma] ; \tau, u) \backslash C \neq \emptyset$ for any $\sigma>\tau$. For instance, if $C$ is the intersection of a finite number of half-planes of the kind $p_{i} \cdot u \leq \beta_{i}(i=1, \ldots, h)$, the same techniques as in [1] (Theorem 16.5) show that $\Gamma^{+}(C) \subseteq \overline{\partial C \backslash K}$, where $K$ is the set of those points $u \in \partial C$ such that, for a.e. $t \in \mathbb{R}$, it is $g(t, u) \cdot p_{i} \leq 0$ whenever $p_{i} \cdot u=\beta_{i}$.

Lemma 6.3. Let $a$ and $V$ be as in Lemma 2.5, and put $W(x)=V\left(x+x_{0}\right), x^{ \pm}=$ $\pm 1-x_{0}$ (so that $\left.x^{-}<0<x^{+}\right), H_{0}(t, x, y)=\frac{1}{2} y^{2}-a(t) W(x)$. Then there exists a function $H(t, x, y)$ such that $H \equiv H_{0}$ for $y \geq 0, H_{1}:=H-H_{0}$ does not depend on $t, H_{1} \in C^{2}\left(\mathbb{R}^{2}\right)$, and the field $g(t, u)=J \nabla_{u} H(t, u)$ enjoys the following property: whenever $u=(x, y)$ solves the equation $\dot{u}=g(t, u)$ and $u( \pm \infty)=\left(x^{ \pm}, 0\right)$, it is $y>0$ everywhere.

Proof. We divide the proof into several steps.

(1) We define $H$ as $H_{0}+H_{1}$, where $H_{1}(x, y)$ takes the form $\frac{1}{3} p(x) \mathrm{min}$ $(0, y)^{3}$, and the function $p$ fulfils the following properties: $p \in C^{2}(\mathbb{R}), p \geq 0$, $p(x)$ is constant for $|x| \leq r_{0}$ (where $\left.r_{0} \in\right] 0, \min \left(-x^{-}, x^{+}\right)$[ is fixed from now on), $p$ is monotone on $\left[x^{-},-r_{0}\right]$ and $\left[r_{0}, x^{+}\right], p(x)=0$ for $x \leq x^{-}$and $x \geq x^{+}$. The constant value $\bar{p}$ on $\left[-r_{0}, r_{0}\right]$ is chosen as follows: since inf $a>0$ and $W^{\prime \prime}(0)=V^{\prime \prime}\left(x_{0}\right)<0$, we can find $m_{0}, M>0$ such that $m_{0}<1$ and

$$
m_{0}|x| \leq a(t)\left|W^{\prime}(x)\right| \leq M|x|, \quad t \in \mathbb{R},|x| \leq r_{0} .
$$

Now, let $0<\omega<\pi / 2, m=m_{0} \cos ^{2} \omega, \lambda>(M+1) /(2 m), 0<h<r_{0} \exp (-\lambda$ $(\pi+\omega)) \sin \omega$ : then we put $\bar{p}=1 / h$. We point out that $H_{1} \in C^{2}\left(\mathbb{R}^{2}\right)$, and the two components $H_{y}^{\prime}$ and $-H_{x}^{\prime}$ of $g=J \nabla_{u} H$ are respectively given, for $y \leq 0$, by $y+p(x) y^{2}$ and $a(t) W^{\prime}(x)-\frac{1}{3} p^{\prime}(x) y^{3}$. We also denote by $g_{i}$ the field 
which correspond to $H_{i}(i=0,1)$, so that $g=g_{0}+g_{1}$. In the same way as $Q_{\lambda}$ corresponds to $g$ in Lemma 6.1, for $i=0,1$ we can associate to $g_{i}$ the function $Q_{\lambda}^{i}$, so that $Q_{\lambda}=Q_{\lambda}^{0}+Q_{\lambda}^{1}$.

(2) According to the notations of Lemma 6.1 , it is $Q_{\lambda} \geq 0$ on $\mathbb{R} \times \Omega^{+}$, where $\Omega^{+}:=\left[-r_{0}, r_{0}\right] \times[0,+\infty[$ and $\lambda>0$ is taken as above. In order to prove this claim, we first show that, whenever $|x| \leq r_{0}, t, y \in \mathbb{R}$ and $u=(x, y)$ :

$$
\begin{aligned}
& \text { (a) } g_{0}(t, u) \cdot J u=y^{2}-a(t) W^{\prime}(x) x \geq m_{0} x^{2}+y^{2}, \\
& \text { (b) } g_{0}(t, u) \cdot u=x y+a(t) W^{\prime}(x) y \geq-m \lambda\|u\|^{2} \\
& \text { (c) } Q_{\lambda}^{0}(t, u) \geq \lambda\left(\left(m_{0}-m\right) x^{2}+(1-m) y^{2}\right) .
\end{aligned}
$$

Indeed, the first inequality of (6.1) easily entails (6.2a) (since $x W^{\prime}(x) \leq 0$ on $\left.\left[x^{-}, x^{+}\right]\right)$, while the second one allows to evaluate from below $g_{0}(t, u) \cdot u$ by $-(M+1)|x y|$ : then $(6.2 \mathrm{~b})$ follows from $2|x y| \leq x^{2}+y^{2}$ and $M+1<2 m \lambda$. Now, in order to get (6.2c), it is enough to multiply (6.2a) by $\lambda$ and add (6.2b). Finally, we easily get our claim from (6.2c), since $m<m_{0}<1$ and $Q_{\lambda}=Q_{\lambda}^{0}$ for $y \geq 0$.

(3) The inequality $Q_{\lambda} \geq 0$ holds on $\mathbb{R} \times D$ as well, where $D$ is a suitable subset of the half-plane $y \leq 0$ : namely, $D$ is the the convex hull of the four points $p_{0}=(0,0), q_{0}=(h / \delta,-h), q_{1}=\left(r_{1},-h\right), p_{1}=\left(r_{1}, 0\right)$, where $\delta=$ $\operatorname{tg} \omega$ and $r_{1}=r_{0} \exp (-\lambda \pi)$ : since $\sin \omega<\delta$ and $h<r_{1} \exp (-\lambda \omega) \sin \omega$, we get, in particular, $h / \delta<r_{1}$, so that $D$ is a right trapezium. Furthermore, $0 \leq x \leq r_{1}$ for any $(x, y) \in D$, so that $p(x) \equiv 1 / h$ and $p^{\prime}(x) \equiv 0$. In particular, $g_{1}(u) \cdot J u=y^{3} / h$ and $g_{1}(u) \cdot u=x y^{2} / h$. Since the latter quantity is non-negative, we argue that $Q_{\lambda}^{1}(u) \geq \lambda y^{3} / h$ : but $-h \leq y \leq 0$, so that, actually, $Q_{\lambda}^{1}(u) \geq-\lambda y^{2}$. Now we can add the last inequality to (6.2c): since $m_{0}=m\left(1+\delta^{2}\right)$, we get $Q_{\lambda}(t, u) \geq \lambda m\left(\delta^{2} x^{2}-y^{2}\right)$, where the last term is non-negative, because $-\delta x \leq y \leq 0$ on $D$.

(4) Let us define $U, \bar{U}_{1}, Q^{-}, Q^{+}$as, respectively: $\left.\left.\left[0, x^{+}\right] \times\right]-\infty,-h\right]$, $\left.\left.\left.\left.\left.\left.\left[r_{1}, x^{+}\right] \times\right]-\infty, 0\right],\right]-\infty, x^{-}\right] \times\right]-\infty, 0\right],\left[x^{-},+\infty[\times[0,+\infty[\right.$. According to the criterion we gave at the end of Def. 6.2, the properties of the function $p(x)$ and also (2.3), we can easily check that $\Gamma^{+}(U)$ and $\Gamma^{+}\left(Q^{-}\right)$are empty, while $\Gamma^{+}\left(Q^{+}\right), \Gamma^{+}\left(U_{1}\right)$ and $\Gamma^{+}(D)$ are respectively contained in $p_{0} x^{+}, p_{1} q_{1}, p_{0} q_{0} \cup$ $q_{0} q_{1}$.

(5) Let $u=(x, y)$ be a solution of the equation $\dot{u}=g(t, u)$ such that $u( \pm \infty)=\left(x^{ \pm}, 0\right)$ : then $y$ is definitively positive (to the right and to the left). Indeed, according to the nature of the equilibrium $\left(x^{-}, 0\right)$, the convergence of $(x(t), y(t))$ to $\left(x^{-}, 0\right)$ is from the left or from the right on both components. Actually, since $\Gamma^{+}\left(Q^{-}\right)=\emptyset$, the former case is ruled out, because $x(+\infty)=$ $x^{+}$. Then $y(t) \rightarrow 0^{+}$as $t \rightarrow-\infty$. In a similar way we can show that $y(t) \rightarrow 0^{+}$ as $t \rightarrow+\infty$, since (2.3) ensures the negative invariance of the set $\left[x^{+},+\infty[\times]-\right.$ $\infty, 0]$.

(6) By contradiction, let $u=(x, y)$ be as in the previous step, but suppose that $y$ is not everywhere positive: we are going to show that $u(t)$ goes into $D$. Indeed, since $y$ is definitively positive to the left, we can consider the first time $\tau_{0}$ at which $y\left(\tau_{0}\right)=0$. But $\Gamma^{+}\left(Q^{+}\right) \subseteq p_{0} x^{+}$, so that $0 \leq x\left(\tau_{0}\right) \leq x^{+}$, and $\left.\left.\left.\left.x(]-\infty, \tau_{0}\right]\right) \supseteq\right] x^{-}, 0\right]$ : then we can find $\sigma_{0}<\tau_{0}$ such that $x\left(\sigma_{0}\right)=-r_{0}$ 
and $y\left(\sigma_{0}\right)>0$. Now we can show that $x\left(\tau_{0}\right) \geq r_{1}$ : of course, since $r_{1}<r_{0}$, it is right to suppose $x\left(\tau_{0}\right) \leq r_{0}$, so that $u(t) \in \Omega^{+}$for $\sigma_{0} \leq t \leq \tau_{0}$. In this case, according to the second step, we can apply Lemma 6.1, by putting in it $\Omega=\Omega^{+}, \sigma=\sigma_{0}$ and $\tau=\tau_{0}$ (so that $0 \leq-\Delta \theta \leq \pi$ ): since $\rho\left(\sigma_{0}\right) \geq\left|x\left(\sigma_{0}\right)\right|=r_{0}$ and $\rho\left(\tau_{0}\right)=x\left(\tau_{0}\right)$ we actually get $x\left(\tau_{0}\right) \geq r_{1}$. Then, after the time $\tau_{0}, u(t)$ reaches $U_{1}$. Now, since $y(t)$ is definitively positive to the right, $u(t)$ must leave $U_{1}$ : but $\Gamma^{+}\left(U_{1}\right) \subseteq p_{1} q_{1}$, so that $u(t)$ enters $D$ at a certain time $\sigma_{1}$.

(7) We show that $u(t) \in U$ definitively. Indeed, what we told about $D$ in the fourth step ensures that, after the time $\sigma_{1}, u(t)$ must cross $p_{0} q_{0}$ or $q_{0} q_{1}$. Let us suppose, for instance, that the former case occurs at a certain time $\tau_{1}$ : then $\rho\left(\tau_{1}\right) \leq \lambda_{0}$, where $\lambda_{0}=h / \sin \omega$ is the length of $p_{0} q_{0}$. According to the third step, let us put $\Omega=D, \sigma=\sigma_{1}$ and $\tau=\tau_{1}$ in Lemma 6.1 (so that $0 \leq-\Delta \theta \leq \omega)$ : we get $\rho\left(\tau_{1}\right) \geq r_{1} \exp (-\lambda \omega)$, so that $r_{1} \leq \lambda_{0} \exp (\lambda \omega)$, in contrast with our choice of $h$. Hence $u(t)$ must cross $q_{0} p_{1}$ and go into $U$ : but $\Gamma^{+}(U)=\emptyset$, so that $u(t)$ does not leave $U$, where $y(t)$ cannot converge to zero.

Proof of Lemma 2.5. We first modify the field $g=J \nabla_{u} H$ of the previous result in such a way that it admits a linear domination, according to Def. 2.3. To this end, we remark that $H(t, x, y)=G(x, y)-a(t) W(x)$, where $G(x, y)=$ $\frac{1}{2} y^{2}+\frac{1}{3} p(x) y^{3}$ for $y \leq 0$ : in particular, since $0 \leq p(x) \leq 1 / h, G(\cdot,-h)$ takes values in the interval $J:=\left[h^{2} / 6, h^{2} / 2\right]$. Now, let $\chi \in C^{2}(\mathbb{R})$ be such that $\chi(s)=s$ for $s$ in an open neighbourhood of $J, \chi^{\prime} \geq 0$ and $\chi^{\prime}$ has compact support: then the function $\tilde{G}(x, y)$ which agrees with $G(x, y)$ for $y \geq-h$ and with $\chi(G(x, y))$ for $y<-h$ is $C^{2}$ as well. Furthermore its gradient is bounded for $y \leq-h$, thanks to the properties of $\chi$, but also for $-h \leq y \leq 0$, since $p$ is bounded in $C^{1}(\mathbb{R})$. On the other hand, $\nabla \tilde{G}(x, y)=(0, y)$ for $y \geq 0$, so that the field $\tilde{g}=J \nabla \tilde{G}$ fulfils a linear domination. Furthermore, thanks to (2.3), the same can be said for $g_{*}(t, x, y)=\tilde{g}(x, y)+\left(0, a(t) W^{\prime}(x)\right)$. We also point out that $g_{*} \equiv g$ for $y \geq-h$, while $\pi_{x} g_{*}=\chi^{\prime}(G) \pi_{x} g$ for $y<-h$, where $\pi_{x}$ denotes the projection on the $x$-axis: in particular, since $\chi^{\prime} \geq 0$, also the positive invariance of the strip $U$ is preserved, when replacing $g$ by $g_{*}$. Now, in order to get the field $f_{*}$ of Lemma 2.5 , we only need to shift $g_{*}$ with respect to $x$, so as to get $f_{*}(t, x, y)=g_{*}\left(t, x-x_{0}, y\right)$, that is to say:

$$
f_{*}(t, x, y)=\left(0, a(t) V^{\prime}(x)\right)+\tilde{g}\left(x-x_{0}, y\right) .
$$

\section{References}

[1] Amann, H.: Ordinary Differential Equations, de Gruyter Studies in Mathematics, vol. 13. Walter de Gruyter, Berlin (1990)

[2] Bonheure, D., Sanchez, L.: Heteroclinic orbits for some classes of second and fourth order differential equations. In: Cañada, A., Drabek, P., Fonda, A. (eds.) Handbook of Differential Equations, vol. III. Elsevier, Amsterdam (2006) 
[3] Brézis, H.: Analyse Fonctionnelle, Collection mathématiques appliquées pour la maitrise. Masson, Paris (1983)

[4] Chen, C.-N., Tzeng, S.-Y.: Existence and multiplicity results for heteroclinic orbits of second order Hamiltonian systems. J. Differ. Equ. 158, 211-250 (1999)

[5] Gavioli, A.: Existence of heteroclinic trajectories for asymptotically autonomous equations. Topol. Methods Nonlinear Anal. 34, 251-266 (2009)

[6] Gavioli, A.: Heteroclinic solutions to asymptotically autonomous equations via continuation methods. Adv. Nonlinear Stud. (to appear)

[7] Gavioli, A., Sanchez, L.: On a class of bounded trajectories for some nonautonomous systems. Math. Nachr. 281(11), 1557-1565 (2008)

[8] Gavioli, A., Sanchez, L.: On Bounded Trajectories for Some Non-Autonomous Systems. In: Differential Equations, Chaos and Variational Problems, vol. 75, pp. 393-404. Birkhauser, Basel (2007)

[9] Hale, J.: Ordinary Differential Equations. Pure and Applied Mathematics, Wiley Interscience, New York (1969)

[10] Rabinowitz, P.H.: Homoclinic and heteroclinic orbits for a class of Hamiltonian systems. Calc. Var. Partial Differ. Equ. 1, 1-36 (1993)

[11] Rabinowitz, P.H., Coti Zelati, V.: Multichain type solutions for Hamiltonian systems. In: Proceedings of the Conference on Nonlinear Differential Equations, vol. 5, pp. 223-235. Southwest Texas State Univ., San Marcos (2000) (electronic)

[12] Spradlin, G.S.: Heteroclinic solutions to an asymptotically autonomous second order equation. Electron. J. Differ. Equ. (to appear)

Andrea Gavioli

Dipartimento di Matematica Pura e Applicata,

Università di Modena e Reggio Emilia,

via Campi 213/B,

41100 Modena,

Italy

e-mail: gavioli@unimore.it

Received: 10 November 2007.

Revised: 01 April 2010.

Accepted: 01 July 2010. 